\title{
Assembly of lamins in vitro
}

\author{
Min Gung Wei, Xiang Jun TONG, Bin Chen, Bo \\ ZHANG, Zhong Fan LiU* Ming Xiao Ding, Zhong \\ HE ZHAI \\ College of Life Sciences, Peking University \\ * College of Chemistry and Molecular Engineering, Peking \\ University Beijing 100871, China.
}

\begin{abstract}
After lamins A, B and C were isolated and purified from rat liver, their assembly properties were examined by electron microscopy and scanning tunneling microscopy using negative staining and the glycerol coating method, respectively. By varying the assembly time or the ionic conditions under which polymerization takes place, we have observed different stages of lamin assembly, which may provide clues on the structure of the $10 \mathrm{~nm}$ lamin filaments. At the first level of structural organization, two lamin polypeptides associate laterally into dimers with the two domains being parallel and in register. At the second level of structural organization, two dimers associate in a half-staggered and antiparallel fashion to form a tetramer $75 \mathrm{~nm}$ in length. At the third level of structural organization, 4-10 lamin tetramers associate laterally in register to form $75 \mathrm{~nm}$ long $10 \mathrm{~nm}$ filaments, which in turn combine head to head into long, fully assembled lamin filaments. The assembled lamin filaments are nonpolar.
\end{abstract}

Key words: Nuclear lamina, lamins, scanning tunneling microscopy, assembly.

\section{INTRODUCTION}

Lamins, being members of the intermediate filament (IF) family[1-3], are components of the nuclear lamina which covers the inner nuclear membrane. The lamina fibers are about $10 \mathrm{~nm}$ in diameter and are arranged in a fibrillar meshwork[4-5]. 
Besides maintaining nuclear envelope integrity[6-7], the lamins may influence interphase chromatin organization, DNA replication and gene expression[8-9].

Lamins are found in a wide range of species. Three lamins (A, B and C) are typically present in mammalian somatic cells. The basic structure of lamin $\mathrm{A}$ and $\mathrm{C}$ are identical except for their C-terminals, perhaps as a result of alternate RNA splicing $[1,2,10]$. In birds, three lamin isoforms have been identified, they are lamins $\mathrm{A}, \mathrm{B}_{1}$ and $\mathrm{B}_{2}[11,12]$ and in Xenopus laevis at least five different lamins have been characterized [13].

Lamin sequences show strong homologies to cytoplasmic intermediate filament proteins[1, 14], and they exhibit three distinct structural domains. They contain a 354-amino-acid-long, conserved central rod domain consisting of four distinct ahelical coils (ie. 1A, 1B, 2A, 2B). Coil 1B has a 42-amino acid extension relative to that of cytoplasmic IF proteins. This central rod domain is flanked by nonhelical $\mathrm{N}$ - and $\mathrm{C}$ - end domains. The lamin N-terminal end domain is small compared with most other intermediate filament proteins (typically 30 residues long), whereas the lamin C-terminal end domain is relatively large (187 to 277 residues long).

It is widely accepted that at the first level of structural organization, two lamin polypeptides associate laterally into dimers with the two domains being parallel and in register[5], thus forming a two-stranded $\alpha$-helical coiled-coil[15, 16]. Electron microscopy of lamin dimers has revealed "myosin-like" molecules consisting of a 50-52 nm long tail being flanked by two globular heads at one end[5, 17]. But how these lamin dimers are arranged within the $10 \mathrm{~nm}$ filaments is not well understood. Analyzing chicken lamin B2 expressed in Escherichia coli, Heitlinger et al.[17] believed that lamin dimers associate longitudinally to form polar head-totail polymers, and these lamin head-to-tail polymers associate laterally into IF-like structures[5]. Aebiet al. also studied lamins extracted from rat liver. They reported that the lamins possess all the major structural properties that have been described for IF polypeptides at the levels both of protomers and of assembled filaments.

In order to study more precisely how $10 \mathrm{~nm}$ lamin filaments are constructed, we extracted and purified lamins A, B and C from rat liver. Electron microscopy (EM) and scanning tunneling microscopy (STM)[18, 19] were employed to investigate their different levels of structural organization.

\section{MATERIALS AND METHODS}

\section{Purification of lamins from rat liver}

Isolation of nuclear envelopes

Nuclear envelopes were isolated from adult rat livers according to Kaufmann[20]. The rat livers were put into STM buffer (250 $\mathrm{m} M$ sucrose, $50 \mathrm{~m} M$ tris-HC1 $\mathrm{pH} 7.4,5 \mathrm{~m} M \mathrm{MgC}_{2}, 1.2 \mathrm{~m} M$ PMSF) immediately after the rats were killed. Then, the livers were minced and homogenized in 4 volumes of the same buffer. After the homogenized sample was filtered through 4 layers cheese cloth and sedimented at $800 \times \mathrm{g}$ for $10 \mathrm{~min}$, the pellet was washed once with STM buffer, then resuspended in DS buffer (2.1 $M$ sucrose, $50 \mathrm{~m} M$ Tris-HC1, pH 7.4, $5 \mathrm{~m} M \mathrm{MgCl}_{2}, 1.2 \mathrm{~m} M$ PMSF). Subsequently, 
the sample was layered over cushions of DS buffer, and sedimented at 70,000 $\times \mathrm{g}$ for $60 \mathrm{~min}$ in a Beckman SW-27 rotor. The pellet (nuclei) was resuspended in STM buffer, layered again over a cushion of DS buffer, and sedimented at $70,000 \times \mathrm{g}$ for $30 \mathrm{~min}$. After the second sedimentation, the nuclei were suspended in STM buffer to a final concentration of $3-5 \times 10^{8} / \mathrm{ml}$, incubated for $60 \mathrm{~min}$ with $250 \mu \mathrm{g} / \mathrm{ml}$ DNase I and $250 \mu \mathrm{g} / \mathrm{ml}$ RNase A, and then sedimented at $800 \times \mathrm{g}$ for 10 min. The nuclei were resuspended at a concentration of $5 \times 10^{8} / \mathrm{ml}$ in LS buffer $\left(0.2 \mathrm{mM} \mathrm{MgCl}{ }_{2}\right.$, $10 \mathrm{~m} M$ Tris-HC1, pH 7.4, $1.2 \mathrm{~m} M$ PMSF), 4 volumes of HS buffer ( $2 \mathrm{~m} M \mathrm{NaC} 1,0.2 \mathrm{~m} M \mathrm{MgCl}_{2}, 10$ $\mathrm{m} M$ Tris-HC1, $\mathrm{pH} 7.4,1.2 \mathrm{~m} M$ PMSF) was immediately added, $\beta$-mercaptoethanol was added to a final concentration of $1 \%(\mathrm{v} / \mathrm{v})$. The samples were incubated for $15 \mathrm{~min}$ and sedimented for $30 \mathrm{~min}$ at $1,600 \times \mathrm{g}$. The extraction with HS buffer was repeated, providing the nuclear envelope fraction.

\section{Purification of nuclear lamin}

The nuclear envelopes were suspended in TM buffer (10\% sucrose, $10 \mathrm{~m} M$ triethanolamine, $\mathrm{pH}$ 7.4, $0.1 \mathrm{mM} \mathrm{MgCl}_{2}, 1 \% \quad \beta$-mercaptoethanol, $\operatorname{lm} M$ PMSF) at a concentration of $2 \mathrm{mg} / \mathrm{ml}$, then an equal volume of $2 \mathrm{M} \mathrm{KC1}$ was added. The samples were layered over a cushion of $30 \%$ sucrose and sedimented at 2,500 rpm for $15 \mathrm{~min}$. The nuclear envelopes were resuspended in TKE1 buffer (10\% sucrose, $2 \%$ Triton X-100, $20 \mathrm{~m} M$ MES-KOH, pH 6.0, $300 \mathrm{~m} M \mathrm{KC} 1,2 \mathrm{~m} M$ EDTA, 1\% $\beta$-mercaptoethanol) at a concentration of $0.25 \mathrm{mg} / \mathrm{ml}$. After incubation at $0{ }^{\circ} \mathrm{C}$ for $30 \mathrm{~min}$ and sedimentation at $6,000 \times \mathrm{g}$ for $20 \mathrm{~min}$, the pellet was resuspended in TKE2 $(2 \%$ Triton X-100, 20 $\mathrm{m} M$ tris-HC1, pH 9.0, $500 \mathrm{~m} M \mathrm{KC1}, 2 \mathrm{mM}$ EDTA, $1 \% \beta$-mercaptoethanol) buffer at a concentration of $0.3 \mathrm{mg} / \mathrm{ml}$. After incubation at $0^{\circ} \mathrm{C}$ for $30 \mathrm{~min}$, the sample was centrifuged at $20,000 \times \mathrm{g}$ for 40 min. The supernatant contained the nuclear lamins.

\section{In vitro assembly of lamins}

The nuclear lamins were dialysed in $25 \mathrm{~m} M$ tris-HC1, $\mathrm{pH}$ 8.0, $250 \mathrm{~m} M \mathrm{NaC} 1,1 \% \quad \beta$-mercaptoethanol ( "equilibration" buffer) for $1 \mathrm{~h}$. Then the product was dialysed in $25 \mathrm{mM} \mathrm{MES,} \mathrm{pH} 6.5,250 \mathrm{mM}$ $\mathrm{NaCl}, 1 \% \beta$-mercaptoethanol ( "polymerization” buffer) for $0.5 \mathrm{~h}$ at room temperature.

\section{Electron microscopy}

Negative staining was used to examine the assembly of lamins in vitro. $5 \mu$ l aliquot of the sample was adsorbed for 1 min to glow-discharged (Aebi and Pollard, 1987), carbon-coated formvar films on copper grids. Then the grid was washed on two drops of distilled water before it was sequentially placed on two drops of $0.5 \%$ uranyl formate for $2 \mathrm{~min}$. Excess liquid was drained with filter paper, before the sample was permitted to air-dry and examined in a JEM-100CX transmission electron microscope.

\section{Scanning tunneling microscopy}

2-3 $\mu$ l of the above sample was directly dropped onto a freshly cleaved, highly oriented pyrolytic graphite (HOPG) surface or gold films without staining or coating with a conductor. After the sample was air dried at room temperature for about $3 \mathrm{~h}$, the graphite was covered with a thin film of $30 \%$ glycerol in order to maintain a definite humidity and a natural environment of the proteins.

Scanning tunneling microscopy was carried out in atmospheric conditions with a Nanoscope III scanning tunneling microscope (Digital Instruments) and a DS-89 STM (Department of Physics, Peking University). In most of the cases, STM was performed in the constant current mode, using a bias voltage of $200 \mathrm{mV}$, and a tunneling current of $0.5 \mathrm{nA}$.

\section{RESULTS}

\section{Isolation and purification of lamins from rat liver nuclear envelopes}

We isolated and purified lamins (include lamin A, B and C) from rat liver nu- 
clear envelopes. The lamins were electrophoresed on an 8\% SDS-polyacrylamide gel which was then stained with Coomassie blue (Fig 1). Two bands were observed, the upper one (68 KD) being lamin A and the other (66 KD) being lamins $\mathrm{B}$ and $\mathrm{C}$ (The molecular weight of the two lamins are similar).

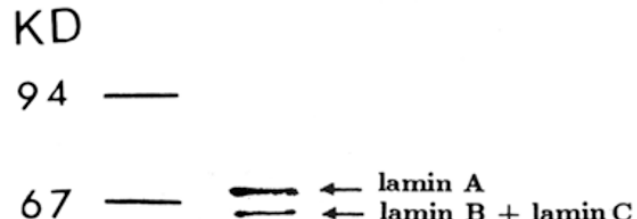

43

30

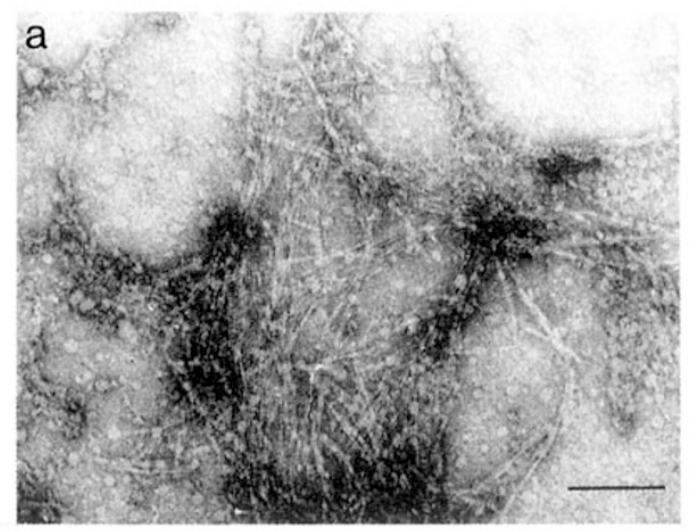

Fig 1. Purified lamins monitored by SDS-PAGE (8\%). Two bands are detected by coomassie blue staining. The upper one (68 $\mathrm{KD})$ is lamin A, the other one with M. W. of $66 \mathrm{KD}$ is lamin B and lamin $\mathrm{C}$.

Fig 2. The purified tamins (lamin A, B and C) from rat liver can self-assemble in vitro. After the sample was negatively stained, the specimen was imaged with a JEM-100CX. In samples assembled for $0.5 \mathrm{~h}$ (a), many filaments with diameter of $10 \mathrm{~nm}$ are visible. After $1 \mathrm{~h}$ of assembly (b), the filaments started to exhibit a characteristic $25 \mathrm{~nm}$ repeat while the diameter of the filaments increased. (a) bar $=150 \mathrm{~nm}$ (b) bar $=100$ $\mathrm{nm}$.

\section{Purified lamins can form filamentous structures in vitro}

Fig 2(a) is an electron micrograph of the negatively stained lamins which had been dialysed against the "polymerization" buffer for about $0.5 \mathrm{~h}$ at room temperature. Lamins have formed filamentous structures with an apparent filament diameter of about $10 \mathrm{~nm}$. After $1 \mathrm{~h}$ of assembly, the filaments started to exhibit a characteristic $25 \mathrm{~nm}$ repeat while the diameter of the filaments increased (Fig 2(b)). 
Min GW et al.

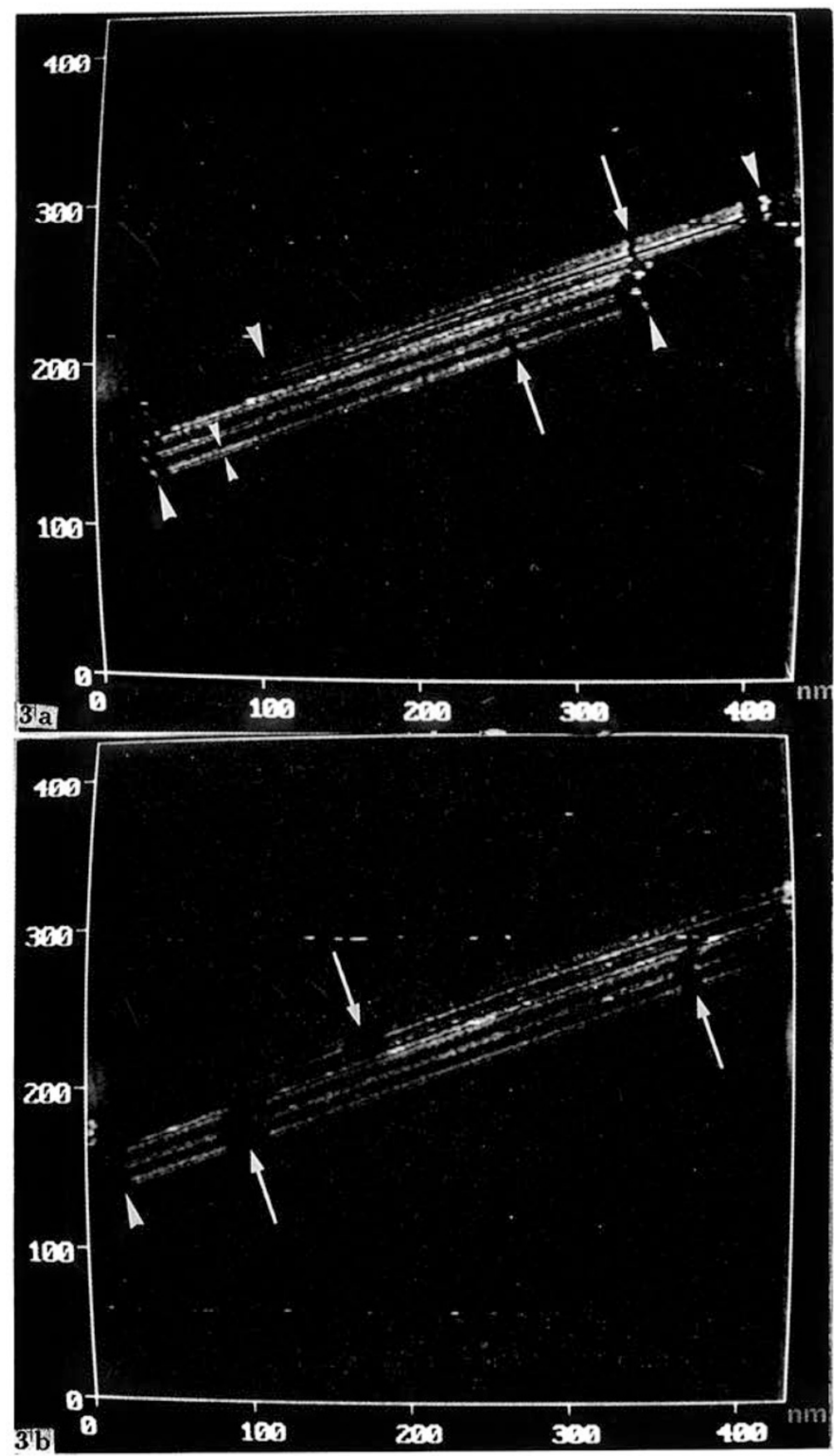

Fig 3. After the purified lamins (including A, B and C) were equilibrated in $25 \mathrm{~m} M$ tris- $\mathrm{HC} 1$, $250 \mathrm{~m} M \mathrm{NaC} 1,1 \% \beta$-mercaptoe-thanol, $1 \mathrm{~m} M$ EGTA, $\mathrm{pH} 8.5$ for $1 \mathrm{~h}$ and incubated at $4^{\circ} \mathrm{C}$ for $12 \mathrm{~h}$, fully assembled lamin filaments can be seen under STM investigation. The filaments are not continuous but are divided into segments by small gaps (as indicated by white arrows). The shortest segments are about $75 \mathrm{~nm}$ in length, while other segments are always a multiple of $75 \mathrm{~nm}$ in length. 
Assembly of lamins in vitro

\section{Lamin filaments are composed of $75 \mathrm{~nm}$ segments}

The purified lamins (including A, B and C) were equilibrated in $25 \mathrm{~m} M$ tris- $\mathrm{HC}$, $250 \mathrm{~m} M \mathrm{NaC} 1,1 \% \beta$-mercaptoethanol, $1 \mathrm{~m} M$ EGTA, $\mathrm{pH} 8.5$ for $1 \mathrm{~h}$ and incubated at $4^{\circ} \mathrm{C}$ for $12 \mathrm{~h}$, before they were dropped onto a newly cleaved graphite surface, which was then examined by scanning tunneling microscopy. Fig 3(a) is a typical image showing a number of fully assembled $10 \mathrm{~nm}$ lamin filaments lying parallel on the graphite surface. The lowest filament (between the two small arrowheads) is about $9.5 \mathrm{~nm}$ in width. The filament lying in the middle looks thinner than the others, because there are two filaments folded together. More interestingly, all the filaments are not continuous, but are divided into two segments by small gaps (as indicated by arrows). The segments to the right of the gaps are about $75 \mathrm{~nm}$ long, which is about 1.5 times the length of a dimer; and the relatively long segments to the left of the gap are $220 \mathrm{~nm}$ long, which is three times as long as $75 \mathrm{~nm}$. In other STM images, we have also observed 10nm fibers of different length, but they are always a multiple of $75 \mathrm{~nm}$; the shortest $10 \mathrm{~nm}$ segments we imaged under the above assembly conditions were $75 \mathrm{~nm}$.

Fig 3(b) is another STM image of the assembled lamins. The shortest segments are also $75 \mathrm{~nm}$ long, but the gaps which separate the short and long segments are different from those in Fig 3(a).

It is also noticed that there are short spherical particles attached to both end of the segments (as indicated by arrowheads). But when two segments are close enough, these spherical particles have disappeared.

In order to avoid any false images, such as the multi-tip effect, we had scanned over the graphite surface for many times at different angles, and found that the images are very stable and identical. So, the STM images reveal the actual structure of lamina filaments. The $75 \mathrm{~nm}$ long segments are important clues to the lamin assembly.

The relative straight alignment of the lamina filaments comparied with those in the electron micrographs is due to the tip sample interactions which can be commonly detected during STM experiments. If the samples are not stably adherent to the substrate, they can be easily moved on the substrate surface resulting in the decreasing of resolution. Only in the places where graphite steps or holes exist, the samples can be steadily imaged. Other sample preparation methods, such as chemical coupling, can also make the sample substrate interactions strong enough for STM. In our STM images of lamina filaments, the graphite steps can be seen especially in the gaps between the filaments. The lamina filaments virtually align alone the graphite step. Because the graphite step are straight, the lamina filaments are straight.

\section{The 75-nm-long segment consists of 4-10 lamin tetramers}

In order to have a close look at the 75-nm-long segments, we then focused the 
Min GW et al.

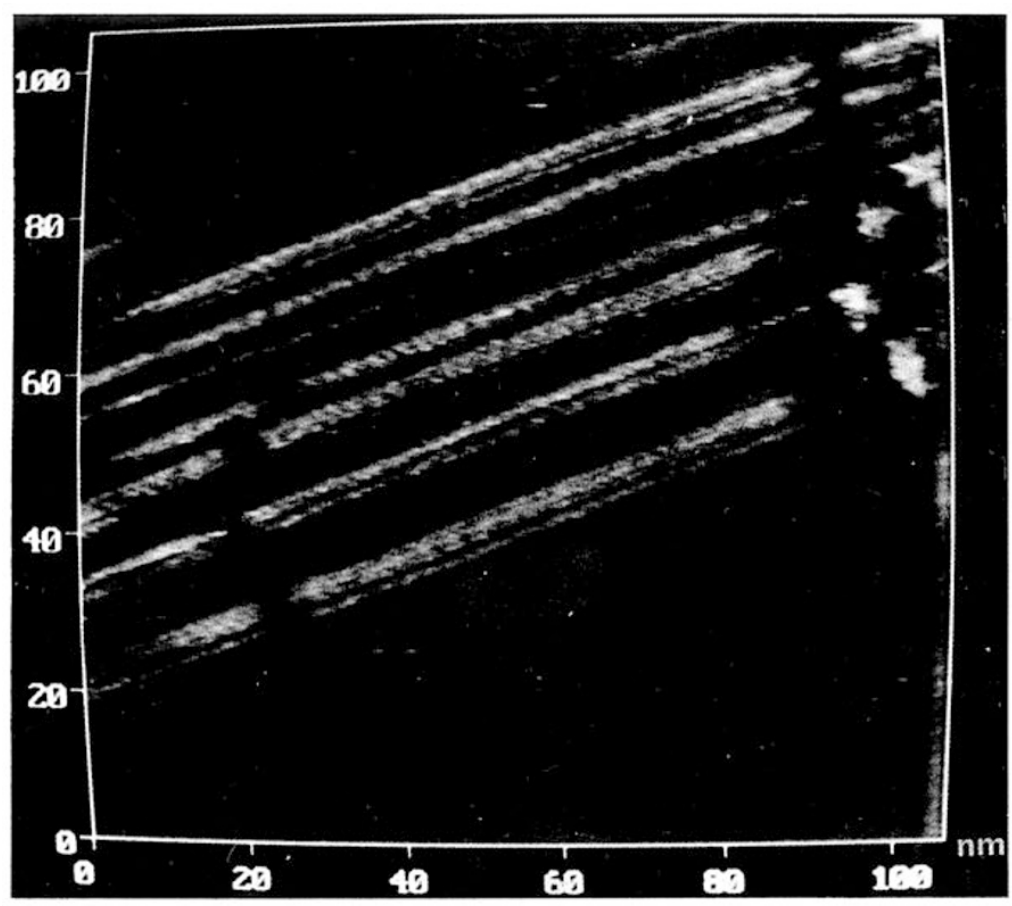

Fig 4. Higher resolution STM image of the 75-nm-long short lamin filaments in the right part of Fig 3(a). Each filament consists of 4-10 thinner filaments, which are known as protofilaments.

Fig 5. Several isolated lamin tetramers lie side by side and head to head on graphite surface. Globular heads are connected to both ends of the tetramers (as indicated by arrowheads). Each of the tetramers is about 75 $\mathrm{nm}$ long and $3 \mathrm{~nm}$ in diameter. bar $=25 \mathrm{~nm}$.

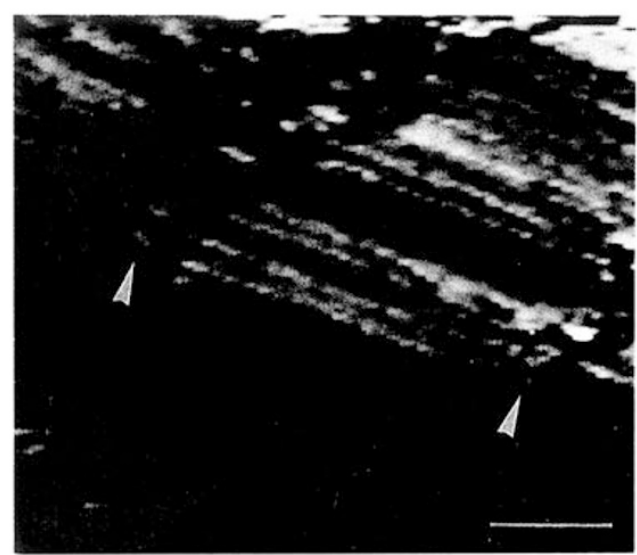

STM tip on a smaller area. Fig 4 is a high magnification view of the right part of Fig 3(a). It is apparent that each $75 \mathrm{~nm}$ long filament segment is composed of 4-10 thinner filaments, which are about $2 \mathrm{~nm}$ in diameter. Most probably, these thin filaments represent the lamin tetramers. The spherical particles (indicated by arrowhead) attached to the filaments are the globular carboxyl- tails of lamins. 
Assembly of lamins in vitro

For rat lamins equilibrated in $25 \mathrm{~m} M$ tris- $\mathrm{HCl}, 250 \mathrm{~m} M \mathrm{NaCl}, 1 \% \beta$-mercaptoethanol, $1 \mathrm{mM}$ EGTA, pH 8.5 for $1 \mathrm{~h}$ and incubated at $4^{\circ} \mathrm{C}$ for $4 \mathrm{~h}$, isolated tetramers can be seen under STM investigation. On a graphite surface, many 2 to $3 \mathrm{~nm} \times 75$ $\mathrm{nm}$ rod-like structures aggregate parallel with spherical particles (indicated by arrowheads) attached to both ends of the rods (Fig 5). Because the length of the rods is longer than that of a lamin dimer, we believe that these rods represent the lamin tetramers. The side by side and head-to-head alignment of the lamin tetramers indicate the interactions not only between the rod domains of the tetramers but also between the head domains.

\section{Lamin dimers observed by scanning tunneling microscope}

STM images (Fig 6) of purified lamins revealed protein molecules with a $50 \mathrm{~nm}$ long rod and a globular head (indicated by arrowheads). The height variation (indicated by arrows) along the molecules demonstrates the coiled coil of the rod domain.

\section{DISCUSSION}

An important property of lamins is that they can spontaneously form filamentous structures in vitro without the help of other cell components or nuclear proteins. After the lamins A, B and C purified from rat liver nuclear envelopes were dialysed against "polymerization" buffer for $0.5 \mathrm{~h}, 10-\mathrm{nm}$-filaments were clearly visible in electron micrographs. These filamentous structures were very similar to those described by Heitlinger et a1.[17]. Furthermore, after $1 \mathrm{~h}$ of assembly, the filaments showed a $25 \mathrm{~nm}$ axial repeat, which is the typical character to all IFs. Presumablely, this repeat may arise from the approximately half staggered structure of lamin tetramers.

By varying the assembly time or the ionic conditions under which polymerization tooks place, different stages of lamin assembly were observed with scanning tunneling microscopy. These structures may provide clues on the molecular architecture of the 10-nm lamin filament (Fig 7).

At the first level of structural organization, two lamin polypeptides associate laterally with their rod domains coiled around each other and their C-terminals at the same end (Fig 7B). The length of a lamin dimer is about $50 \mathrm{~nm}$. That only one head is visible in the STM images may have two reasons. One is the orientation of the dimers, the two heads may fold together, and STM can only follow the surface feature of samples, so only one head is visible. The other reason is that different types of lamins may arrange differently within a dimer. It has been found that lamin $\mathrm{A}$ and $\mathrm{C}$ extracted from rat liver form a dimer with two globular heads, but lamin B dimer have only one head[5].

At the second level of structural organization, we believe that two lamin dimers associate together to form a tetramer (Fig 7C). The existence of lamin tetramers has also been demonstrated previously[5, 22]. It is assumed that during mitosis, lamin 
Min GW et al.
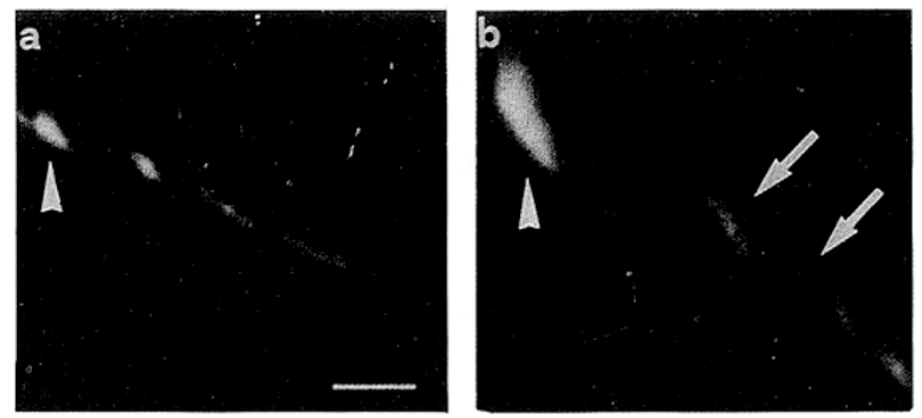

Fig 6. STM images of purified lamins reveals a protein molecule contains a $50 \mathrm{~nm}$ long coiled coil rod (indicated by arrow) and a globular head (indicated by arrowheads). Fig b is a magnified image of Fig a. Bar=10 $\mathrm{nm}$.

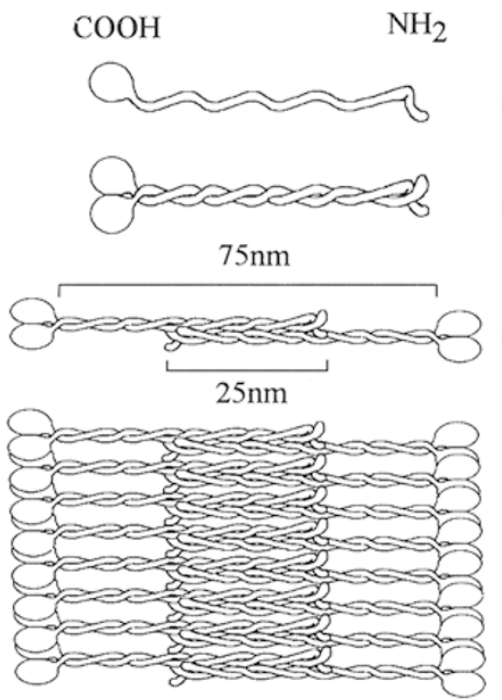

(A)

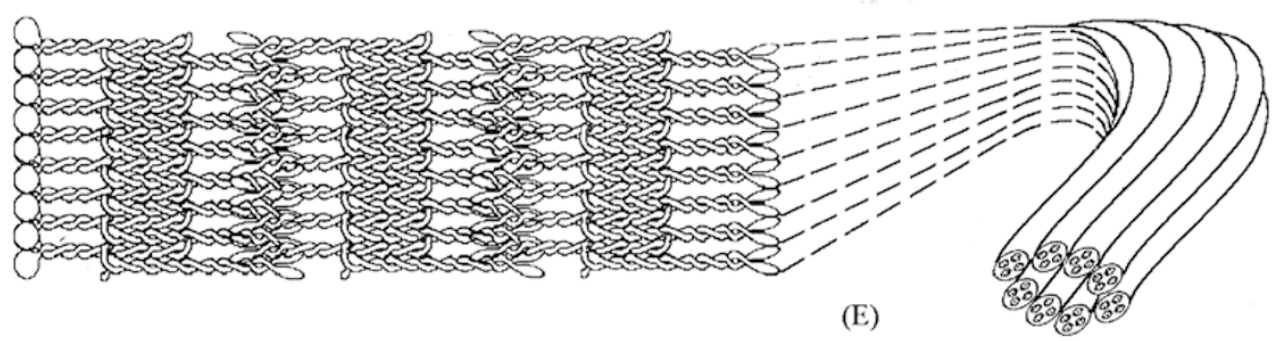

Fig 7. The assembly model according to the STM images. (A) lamin monomer with 50 $\mathrm{nm}$ long rod domain and a global head. (B) two lamin polypeptides lie side by side with their rod domains wound together and their C-terminal at the same side. (C) two lamin dimers associate laterally in a half staggered and antiparallel manner to form a $75 \mathrm{~nm}$ by $3 \mathrm{~nm}$ protofilament. (D-E) several lamin protofilaments associate laterally in register to form 10-nm-width short rod like segments, which in turn link longitudinally to form long lamin filaments. And the assembly of these short segments is nonpolar. 
$\mathrm{A}$ and $\mathrm{C}$ in mammalian cells are dissociated into dimers and tetramers after lamina depolymerization. And in vitro conditions, Aebi et al.[5] had used sedimentation equilibrium method and showed that lamin dimers can further associate into tetramers in a concentration-dependent fashion.

According to the STM images, we can conclude that two lamin dimers aggregate together in a half staggered and antiparallel manner within a tetramer. The length of the tetramer indicates that the two dimers do not fully overlap or associate headto-tail, but are instead assemble into pairs in a staggered fashion, so that within a pair one dimer is axially displaced by half its length $(25 \mathrm{~nm})$ with respect to its neighbor.

The proof of antiparallel orientation of dimers within a tetramer comes from the morphology of isolated tetramers. In Fig 5, there are globular heads attached to both ends of the tetramers, which indicates that the two dimers associate with their rods coiled together and the two heads in the opposite direction. The nonpolar assembly of the 75-nm-long segments also suggests the antiparallel structure.

At the third level of structure organization, several (4-10) lamin protofilaments associate laterally in register to form the 10-nm-thick filament segments, which in turn link head to head to form long lamin filaments (Fig $7 \mathrm{D}$ and E). Because there are head groups at both ends of the filaments in Fig 3(a), the assembly of these filaments is apolar. The ability of lamin tetramers to aggregate laterally to form stable structures is strongly supported by the side by side alignment of lamin tetramers in Fig 5.

The shortest 10-nm-filament also supports our opinions. In the assembly of vimentins, Ip et a1.[23] also found $66 \mathrm{~nm}$ long intermediate filament. They called it the "minimal length" IF, and deduced from it that IF are staggered in some way. The difference is that they thought the stagger occurs between tetramers, but our results demonstrate the staggered structure within the tetramers.

The different length of the segments and the different width of the gaps suggested that the filaments were assembling. Although we can't see the dynamic processes of the lamin assembly, it is reasonable to picture it as follows: the minimal length lamin filaments first form under the interactions of tetramer rod domains, and then when two short filaments are close enough, the interactions of the end groups cause them to come closer. The gap between the two filaments becomes smaller and smaller until the two filaments are joined together.

The head groups in the minimal length filaments obviously play an important role in the assembly processes. But the exact functions and the structural changes of it during assembly are not clearly understood now. It is assumed that the coiled $a$-helices of lamin polypeptides are necessary for the assembly of lamins, while the C-terminals are relevant for the connection of lamins with the nuclear membrane. Under in vitro conditions, Moir et al.[24] found that the deletion of the N- or Cterminal non-helical domains altered the solubility and aggregation properties of lamins, indicating that both domains have a role in lamin assembly. 
Although in our experiments, we can not tell how the head groups influence the assembly of lamins, it is definite that the disappearance of head groups is not caused by their loss. The changes in diameter and the distance between heads and rod domains both indicate the occurrence structural changes during assembly processes.

\section{REFERENCES}

[1] McKeon FD, Kirschner MW, Caput D. Homologies in both primary and secondary structure between nuclear envelope and intermediate filament proteins. Nature 1986; 319:463-8.

[2] Fisher DZ, Chaudhary N, Blobel G. cDNA sequencing of nuclear lamins A and C reveals primary and secondary structural homology to intermediate filament proteins. Proc Natl Acad Sci USA. 1986; 83: 6450-4.

[3] Franke WW. Nuclear lamins and cytoplasmic intermediate filament proteins: a growing multigene family. Cell 1987; 48:3-4.

[4] Gerace LA, Blum A, Blobel G. Immunoeytochemical localization of the major polypeptides of the nuclear pore complex-lamina fraction. J Cell Biol 1978; 79:546-66.

[5] Aebi U, Cohn J, Buhle L, Gerace L. The nuclear lamina is a meshwork of intermediate-type filaments. Nature 1986; 323:560-4.

[6] Newport JW, Wilson KL, Dunphy WG. A lamin-independent pathway for nuclear envelope assembly. J Cell Biol 1990; 111:2247-59.

[7] Whytock S, Moir RD, Stewart M. Selective digestion of nuclear envelopes from Xenopus oocyte germinal vesicles: possible structure role for the nuclear lamina. J Cell Sci 1990; 97:571-80.

[8] Lebkowski JS, Laemmli UK. Non-histone proteins and long-range organization of Hela interphase DNA. J Molec Biol 1982; 156:325-44.

[9] Benevente R, Krohne G. Involvement of nuclear lamins in postmitotie reorganization of chromatin as demonstrated by microinjection of lamin antibodies. J Cell Biol 1986; 94:1847-54.

[10] Riedel W, Werner D. Nucleotide sequence of the full-length mouse lamin C cDNA and its deduced amino-acid sequence. Biochem Biophys Acta 1989; 1008:119-22.

[11] Vorburger K, Lehner CF, Kitten GT, Eppenberger HM, Nigg EA. A second higher vertebrate B-type lamin: cDNA sequence determination and in vitro processing of chicken lamin B2. J Mol Biol 1989; 208:405-15.

[12] Peter M, Kitten GT, Lehner CF, et al. Cloning and sequencing of cDNA clones encoding chicken lamins A and B1 and comparison of the primary structures of vertebrate A- and B-type lamins. J Mol Biol 1989; 208:393-404.

[13] Stick R. cDNA cloning of the developmentally regulated lamin L-III of Xenuopus laevis. EMBO J 1988; 7:3189-97.

[14] McKeon FD. Nuclear lamin proteins: Domains required for nuclear targeting assembly and cell-cycle-regulated dynamics. Curr Opin Cell Biol 1991; 3:82-6.

[15] Parry DAD, Conway JF, Steinert PM. Structural studies on lamins: Similarities and differences between lamin and intermediate-filament proteins. Biochem J 1986; 238:305-8.

[16] Krohne G, Wolin SL, McKeon FD, Franke WW, Kirsehner MW. Nuclear lamin L1 of Xenuopus laevis: cDNA cloning amino acid sequence and binding specificity of a member of lamin $\mathrm{B}$ subfamily. EMBO J 1987; 6:3801-3808.

[17] Heitlinger E, Peter M, Haner M, Lustig A, Aebi U, Nigg EA. Expression of chicken lamin B2 in E.coli: Characterization of its structure assembly and molecular interactions. J Cell Biol 1991; 113:485-95.

[18] Driscoll RJ, Youngquist MG, Baldeschwieler D. Atomic-scale imaging of DNA using scanning tunneling microscopy. Nature 1990; 346:294-6. 


\section{Assembly of lamins in vitro}

[19] Lindsay SM, Lyubchenko YL, Tao NJ, et al. STM and AFM studies of biomaterials at a liquid-solid interface. J Vac Sci Technol 1994; All (4):808-15.

[20] Kaufmann SH, Gibson W, Shaper JH. Characterization of the major polypeptides of the rat liver nuclear envelope. J Biol Chem 1983; 258:2710-9.

[21] Aebi U, Pollard TD. A glow discharge unit to render electron microscope grids and other surfaces hydrophilic. J Electron Microsc 1987; 7:29-33.

[22] Gerace LA, Blobel G. The nuclear envelope lamina is reversibly depolymerized during mtosis. Cell 1980; 19:277-87.

[23] Ip W, Hartzer MK, Pang YYS, Robson RM. Assembly of vimentin in vitro and its implications concerning the structure of intermediate filaments. J Mol Biol 1985; 183:365-75.

[24] Moir RD, Donaldson AD, Stewart M. Expression in Escherichia coli of human lamins A and C: influence of head. J Cell Sci 1991; 99:363-72.

Received 8-12-1995. Revised 20-5-1996. Accepted 22-5-1996. 